\title{
Meaning Construction of Internet News Discourse from the Perspective of Multimodal Discourse Analysis
}

\author{
Yan Liu \\ Tianjin University Renai College \\ Tianjin, China
}

\begin{abstract}
Nowadays internet has become the fourth media in the information age and plays an indispensable role in acquiring information and the latest news. Being a new massmedia, internet news is reported with an increasing number of sounds, colors, images and videos to convey the information more vividly and effectively. In fact, the construction of meaning in news discourse depends on both language and other modes. The former researches on news discourse are confined to linguistic features, which are not appropriate for analyzing internet news. Based on Halliday's Systemic Functional Grammar, Kress and Van Leeuwen put forward Visual Grammar, exploring the meanings of discourse from the multimodal perspective, which provide us a new method to analyze discourses. The visual and verbal semiotic modes in internet news are two different resources of meaning, which work together to construct the meaning. This paper will conduct a comprehensive study on the meaning construction of news discourses and offer readers a new perspective for interpreting the meaning.
\end{abstract}

Keywords—news discourse; meaning construction; visual grammar; multimodal discourse analysis

\section{INTRODUCTION}

Along with the dramatic changes and improvements in science and technology, people's way of communication changed from single-modality to multimodal mode. The development of multimedia technology helps people get access to more information. The popularization of internet technology brings us into a new era of acquiring information and people get to know the world more conveniently. The application of non-verbal symbols in internet, such as colors, voices and images makes the news more colorful and efficient. The majority of people gets used to reading the news from the internet and knows what is happening in the world. Internet news is published in the form of words, pictures and videos so as to be more vivid and eye-catching. In fact, the meaning is seldom conveyed by the verbal semiotic alone and the visual text plays a more important role in decoding the meaning of news. The multimodal symbols such as the sound, the image, the color and the typography are potential meaning resources. Therefore, it is necessary to analyze the news from the perspective of multimodal discourse analysis to understand the news comprehensively and correctly.

\section{PREVIOUS STUdIES ON NEWS Discourse}

\section{A. Definition of News}

News refers to the report of the recent event, which is written by the journalist for the purpose of informing the readers the latest occurring events. O'brien (1918) puts forward an impressive definition of news. NEWS represents for everything that happens to the north, east, west and south part of the world, it also means something new happened in the world. This definition tends to be more humorous and less academic, but it does give us a clear vision about the basic features of news, such as freshness, significance, attractiveness and prominence.

\section{B. Features of Internet News}

Besides the fundamental features of news such as, timely, informative and authoritative, the remarkable characteristic of internet news is the combination of verbal modality and visual modality. In fact, pictures and words are commonly used in internet news. The interaction between them constructs the meaning in a particular way. Words describe the implied meaning of the pictures. Pictures transmit the information of the words and impact viewers by means of conceptual processes, social distance, attitude, modality, salience, framing and so on. Words and pictures compose the news discourse together, which are complementary to each other.

\section{Discourse Analysis of News}

News discourse has aroused an increasing attention of scholars abroad and at home. Many branches of scholars attempt to study it from different perspectives such as content analysis, structural analysis, critical discourse analysis, systemic functional linguistics, which contribute a lot to the development of news discourse analysis. It is far from enough to explore the deep meaning of internet news. Besides language, other symbolic forms such as sounds, images and colors play an indispensable role in the construction of meaning in internet news. Therefore this thesis will make a further study on the internet news discourse from the perspective of multimodal discourse analysis so as to interpret the meaning accurately. 


\section{THEORETICAL FRAMEWORK FOR ANALYZING MULTIMODAL DisCOURSE}

During the process of analyzing multimodal discourse, a large number of scholars come to realize that it is impossible as well as not convincing to conduct discourse analysis only by studying the linguistic factors. Non-linguistic factors also play an important role in expressing and constructing the whole meaning of the discourse. It is widely accepted that discourse analysis has to shift from monomodality to multimodality, which has offered scholars a brand new method to promote their awareness and capacity of decoding the meaning by means of exploring different modes.

\section{A. Halliday's Systemic Functional Grammar}

Halliday views language as a kind of semiotic mode and puts forward systemic functional grammar (SFG), setting up a flexible approach for the discourse studies. Halliday proposes the concept of meta-functions, which includes the ideational function, the interpersonal function and the textual function. According to Halliday, the ideational metafunction can be viewed as the meaning of content, reflecting our experience and understanding of the world. The experience of the producer can be represented by transitivity system no matter it is real or imaginary. The interpersonal metafunction stresses on the construction of social relationships. In other words, it means to socialize with others by the use of language, showing the author's opinion and influencing the viewer's attitude. The textual metafunction deals with the problem of internal organization of a text, emphasizing on the cohesion and coherence of a text.

\section{B. Kress and Van Leeuwen's Visual Grammar}

Based on Halliday's systemic functional grammar, Kress and Van Leeuwen put forward visual grammar. The innovative point of visual grammar is that visual image can be interpreted as text; the way to decode the visual image can be view as the grammar to study the visuals. Here grammar represents a set of social resources for the construction of meaning instead of a set of rules for the construction of language. They point out that the visual designs, such as language and any other semiotic modes, can meet the requirements for the representation of the reality world, for the interaction between the producer and the receiver, for the compositional arrangements of visual resources. They introduce three components in visual grammar. They are representational resources, interactive resources and composition. Representational resources, such as the participants, events and objects, can represent the material world visually. An interactive resource refers to the interaction between the viewers and what is viewed. Composition refers to the information value or the relative emphasis on the text and image. Visual grammar provides us a reliable theoretical perspective to analyze the internet news.

\section{Multimodal Discourse Analysis}

Lim is a prominent scholar in studying the relationships between language and visual images in a multimodal discourse. Lim introduces the Integrative Multisemiotic Model and attempts to analyze the text from the aspects of expression plane, content plane and context plane. In IMM, language and images are communicative modes, sharing ideology, genre, register, and utterance meaning. MDA has not been introduced to China until 2003, from then on many Chinese scholars show great interest in this field. Zhang Delu is one prominent scholar in this field. He set up a theoretical framework for MDA on the basis of SFL. He researches the multimodal discourse systems from five levels, namely, context of culture, context of situation, semantic level, formal level and media level. What's more, he pays attention to the relationship among various modalities and the construction of grammar in multimodal discourse. Scholars have achieved remarkable achievement on the study of MDA in theoretical framework as well as in practical application. However there are still some limitations in the integration of visual and verbal modalities in news discourse. Therefore, it is advisable to further the study from the perspective of multimodal discourse analysis.

\section{Meaning Construction of Multimodal NeWs DISCOURSE}

Internet has changed our ways of living and people prefer to acquire the information from the internet to make good use of fragmented time than read long and unbroken text. Because of internet technology, people receive more pictures, videos and even live shows than ever before and get more involved in multimodal discourses. So it is unavoidable to follow the trend to interpret the meaning by analyzing the semiotic resources. Multimodal discourse is known as a type of discourse in which two or more than two semiotic modes interact with each other to construct meaning. In fact, almost all discourses can be viewed as multimodal discourse. The meaning of multimodal discourse is constructed by language as well as by other modes, such as image, sound, distance, gesture and color. As a result, the academic research on discourses has changed dramatically. Scholars from linguistics, semiotics and other fields realize that language is no longer the sole method to convey meaning. This thesis takes a piece of news from China Daily as an example to illustrate the construction of meaning in internet news.

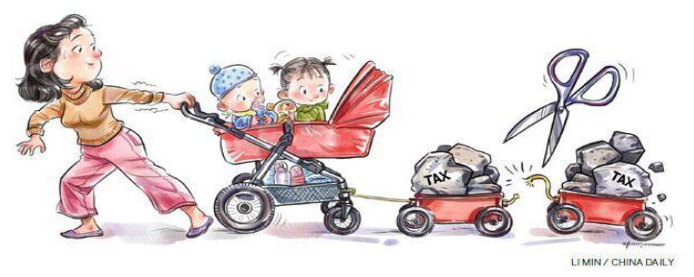

Fig. 1. Government considers ways to build a 'parent-friendly environment'.

\section{A. Meaning Construction through Words}

Headline is the most important part of news, especially for the internet news, because the reader can only read the headline on the homepage. The headline can help the reader decide whether to click the news and read the whole article. Although more and more news are reported in multimodal forms, the role of words shouldn't be neglected. When readers read the headline, Government considers ways to 
build a 'parent-friendly environment', they may find out that parent-friendly environment has been emphasized by quotation marks. Therefore they want to know "What is parent-friendly environment?" and "What might be the ways to achieve the parent-friendly environment?" In order to satisfy their curiosity, readers will continue to read the whole article. From the body part of the news they know that government plans to raise the personal income tax threshold and introduce expense deductions for certain items involving children's education and treatment for serious illnesses in order to encourage more parents to give a birth to the second child. Meaning construction through words is the basic and necessary part of internet news.

\section{B. Meaning Construction through Pictures}

In the age of new mass-media, language itself can't meet people's communicative needs. It is advisable to understand the meaning of a specific text both by analyzing the semiotic resource of language and other semiotic resources. Kress and Van Leeuwen's Visual Grammar provides a good choice to decode the visual meaning.

1) Representational meaning: According to Kress and Van Leeuwen, representational meaning can be divided into two types, one is narrative and the other is conceptual. The distinction between narrative and conceptual structures lies in the presence of a vector.The image bearing a vector can be defined as narrative while the image without a vector can be defined as conceptual. In narrative representation, there are action process, reaction process, verbal and mental process. The conceptual structures also can be divided into three types, i.e., classificational process, analytical process, and symbolic process. To have a further understanding about this concept, it is necessary to distinguish two terms, represented participants and interactive participants. Represented participants refers to people, places and things appearing in images, while interactive participants are the producers and viewers of images, who communicate with each other through images.

This piece of news belongs to narrative representation because the vector has been realized by an oblique line ranging from the eyes of the mother to the stones on the cart. The represented participants are the mother and the two babies. The focus of the eyes, the scissors and the stones on the carts interact with the reader, which are the interactive participants. The former part of the picture is mother and babies, representing for the happiness of life, while the later part is the tax stones on the cart, representing the burden of life. The contrastive colors between the persons and the tax also help the readers decode the deep meaning of the news.

2) Interactive meaning: In addition to the interactions between the people and things appearing in images, visual communication has another resource for constituting interaction between the producer and the viewer of the image, which are contact, social distance, and perspective. Contact refers to eye contact between the participants and viewers. For example, the participants may gaze at the viewers to demand something from them or to offer information to them. Social distance determines the distance we keep from one another. The length of the distance is determined by the intimacy between the participants and viewers, therefore it is a kind of social relation. Perspective means the viewers' attitude toward the image participants, usually represented by visual angle. For instance, looking down perspective means a higher social status; looking up perspective means the viewers have a lower social status, while looking at eye level means it is an equal relation between the participants and the viewers.

The mother is gazing at the tax stones, which shows the demand of reducing the taxes. The mother in this picture is close to the viewer, which means she has a intimate relationship with the viewers. The surprise expression of the babies represents that reducing taxes is out of people's expectation and people feel happy about this.

3) Compositional meaning: Compositional meaning refers to the way of organizing the representational meaning and interactive meaning so as to form a meaningful whole. The compositional meanings can be achieved by three interrelated systems. They are information value, salience, and framing. The information value is usually achieved by the placement of the specific element. Salience focuses on how to highlight some elements over others by utilizing various factors, such as color contrasts, placement of the represented participant, perspective and so on. As for framing, it means the visual elements can be viewed as a whole or separated parts. There are different ways to show the connection of visual elements, such as the absence of framing devices, the similarities of color. The disconnection of elements usually be presented through frame lines, the empty space or contrastive colors. Framing normally makes a sense of detachment between the visual and the viewer, leading to a feeling of objectivity and unemotional. The absence of frame can create a sense of closeness, inviting the readers into the image.

The information value is achieved by the appearance of the babies, which tells the viewer that the realistic meaning of reducing taxes is to encourage the parents to raise two children. Salience is embodied by the extremely large scissors, which can reflect the determination of the government. Framing is reflected by the contrastive colors of the persons and the taxes. Framing is realized by the space between the babies and the cart, which means the taxes shouldn't affect people's happy life.

\section{Meaning Construction through Context}

Context refers to the environment in which the language is used. Zhang Delu, a famous Chinese linguist, points out that communication is influenced by contextual factors. As an important social activity, news is usually reported under certain contexts, especially the social context, therefore it is necessary to interpret the meaning by applying the concept of context. A 'parent-friendly environment' can show the situational context of the news: the birthrate is low in China 
and people get reluctant to have the second child because of the high cost of raising a child. Therefore government wants to build a 'parent-friendly environment' by raising the personal income tax threshold and introducing expense deductions to reduce parents' burden.

\section{Meaning Construction through Culture}

Zhang Delu holds the view that it is the cultural level that makes multimodal communication possible. In fact, cultural level is an important factor in constructing multimodal meaning. Without the cultural level, situational context will lose its explanatory power. Culture is formed on the basis of thinking patterns, life habits and ideology. Language is a kind of cultural phenomenon and it is the carrier of culture. On the one hand, the connotation of culture can be reflected by the language, on the other hand, language cannot be expressed or understood clearly without the cultural background. News belongs to language and is the ideological symbol, which can reflect the cultural heritage of one nation. The headline of this news may lead the viewers to explore the accurate meaning of 'parent-friendly environment', but it is far from enough to decode the meaning from lexical level. In fact Chinese culture is reflected in this news, so it is compulsory to take the culture into consideration to analyze the meaning. It is a tradition in China to raise several children in the past and there is a famous Chinese saying, more children, more blessing. Parent is the corresponding word to child, when we mention parents, we will think about children. Thus the headline indicates that government plans to create a good environment for parents to raise more children. Take a clear look at the picture, we can see there are a boy and a girl in the baby stroller because Chinese people tend to have a son and a daughter in one family. The Chinese character 好 (perfect) is the best illustration of this concept because it is formed by 子(a son) and 女(a daughter).

\section{CONCLUSION}

Internet news, as a new medium of spreading the information, has its own characteristics, such as timely, fresh, convenient, colorful and multimodal. Previous studies of discourse analysis focus on content, structure and genre, overemphasizing language itself and neglecting the function of visual mode. Inspired by Halliday's SFG, Kress and Van Leeuwen's Visual Grammar and Zhang Delu's five levels in MDA, this thesis is aimed at revealing how the multiple modes interact with each other to construct the meaning. This thesis takes Government considers ways to build a 'parent-friendly environment' as an example and makes a further explanation on meaning construction of internet news discourse from the perspective of words, pictures, context and culture. The application of MDA provides a more comprehensive perspective for discourse analysis. It is expected that more and more scholars could break the restrictions of traditional discourse analysis and have a deep insight on discourse analysis.

\section{ACKNOWLEDGEMENT}

This article is the staged achievement of Research on Blended Learning under the Background of Internet Plus from Ministry of education, educational management information center (Project number:EIJYB2017-027). Meanwhile this article is the research achievement of Tianjin universities and colleges innovation project: Tianjin University Renai College Teaching Research Project (Project number: 2017-1-4).

\section{REFERENCES}

[1] Fowler, R, "Language in the news: discourse and ideology in the press,” London\&New York: Routledge, 1991.

[2] Halliday, M.A.K, “An introduction to functional grammar," London: Edward Arnold, 1994.

[3] Halliday, M.A.K, "Language as social semiotic: the social interpretation of language and meaning," London: Edward Arnold,1978.

[4] $\mathrm{Hu}$ Zhuanglin, "Multimodalization in social semiotics," Beijing:Foreign Language Teaching and Research, 2007

[5] Kress, G.\&T.Van Leeuwen, "Multimodal discourse: the modes and media of contemporary communication," London: Arnold, 2001.

[6] Kress, G.\&T. Van Leeuwen, "Reading images: the Grammar of visual design,” London: Routledge, 2006.

[7] Li Zhanzi, "Social semiotic approach to multimodal discourse analysis," Nanjing:Foreign Languages Research,2003

[8] Lim, F.V, "Developing an integrative multi-semiotic model[A]. in kay O'Halloran(eds) multimodal discourse analysis: systemic functional perspectives," London and New York: Continuum, 2004.

[9] Norris, S, "Analyzing multimodal interaction: a methodological framework," London:Routledge, 2004.

[10] Zhang Delu, "On a synthetic theoretical framework for multimodal discourse analysis,” Beijing: Foreign Languages in China, 2009 